
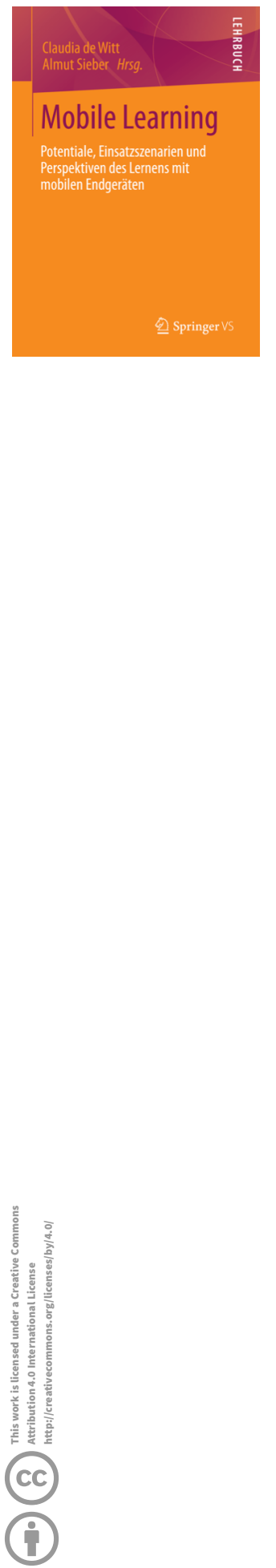

Claudia de Witt, Almut Sieber (Hrsg.)

Mobile Learning: Potenziale, Einsatzszenarien und Perspektiven des Lernens mit mobilen Endgeräten

Wiesbaden: Springer VS, 2013. 278 Seiten

ISBN: 978-3-531-19483-7

$€$ 39.99; CHF 53.90

\section{Mobile Learning}

Die Herausgeberinnen des Sammelbands fokussieren das Thema Mobiles Lernen mit digitalen Endgeräten, welches bereits in Hochschulen und in Unternehmen Einzug gehalten hat. Dennoch wird das Lernen mit mobilen Endgeräten vielerorts als neues Thema identifiziert, mögliche Einsatzszenarien sind oftmals unbekannt. Zumeist wird mit dem mobilen Lernen das schnelle Nachschlagen von Informationen oder die Nutzung von Applikationen z.B. zur geografischen Navigation assoziiert. Mobiles Lernen bietet jedoch ein deutlich vielfältigeres Spektrum. So können informelle Lernprozesse im Kontext des lebenslangen Lernens realisiert sowie Ad-hoc-Bedürfnisse befriedigt werden. Als Problem wird die Unkenntnis hinsichtlich des technischen Aufwandes wie der Auswahl und Anwendung spezieller Software bzw. notwendiger Programmierkenntnisse wahrgenommen. Darüber hinaus fehlt es häufig an Ideen zur Realisierung didaktischer Szenarien.

Frau Prof. de Witt von der FernUniversität in Hagen leitet die Forschungsgruppe Mobile Learning am Lehrgebiet Bildungstheorie und Medienpädagogik, die sich seit längerer Zeit mit dem Thema auseinandersetzt. Seit sechs Jahren wird regelmässig der «Mobile Learning Day» veranstaltet. Die Bandbreite der Referenten ist gross, neben Fachvorträgen aus Hochschulen werden auch Beiträge aus Unternehmen präsentiert. Kennzeichnend für den Mobile Learning Day sind Einblicke in konkrete Anwendungsszenarien und bereits realisierte Projekte. Im vorliegenden Sammelband stellen de Witt und Sieber Ergebnisse aus der eigenen Forschungsgruppe sowie ausgewählte Vorträge des Mobile Learning Day vor.

Der Sammelband umfasst drei Teile und gibt einen Überblick sowohl über den aktuellen fachwissenschaftlichen Diskurs als auch über interessante Beispiele aus der Praxis. Im Mittelpunkt des ersten Teils stehen Aspekte wie Lernen, Arbeiten und Forschen mit Mobile Learning, im zweiten und dritten 
Teil wird nach Anwendungskontexten in Hochschulen und Unternehmen differenziert.

\section{Teil 1: Lernen, Arbeiten und Forschen mit Mobile Learning}

Im Gegensatz zum E-Learning, das sich in Wissenschaft und Praxis seit vielen Jahren als Thema etabliert hat, hat die Beschäftigung mit Mobile Learning deutlich später eingesetzt. De Witt erläutert im ersten Beitrag die Besonderheiten und Potenziale des Mobile Learning. Aufgrund der wachsenden Selbstverständlichkeit einer ubiquitären Verfügbarkeit von Informationen und Wissen auf mobilen Endgeräten wird eine zunehmende Akzeptanz der Nutzung mobiler Endgeräte sowohl im Wirtschafts- als auch im Privatleben festgestellt. Diese Chance nutzen Arbeitgeber, um situative und kontextbezogene Inhalte für Lern- und Arbeitssituationen zu entwickeln. Freie Zeiten können zu Lernzeiten genutzt werden, aktuelle Aufgaben und Herausforderungen können interaktiv bearbeitet werden. Mobiles Lernen unterstützt laut de Witt die Konvergenz von Lernen und Arbeiten, Expertenwissen entwickle sich zu «shared knowledge». Die neuen Formen des lebenslangen, eigenverantwortlichen und selbst organisierten Lernens begünstigen allerdings auch die Entgrenzung von Berufs- und Privatleben. Die Autorin weist darauf hin, dass die Herausforderung darin bestehe, das Thema Mobile Learning in umfassendere Bildungsangebote $\mathrm{zu}$ integrieren und mobile Anwendungen nicht als Einzellösung anzubieten.

Die Autorin des zweiten Beitrags, Judith Seipold, konzentriert sich auf die theoretischen Hintergründe von Mobile Learning. Da der Wissenschaftsprozess der Mobile-Learning-Diskussion im deutschsprachigen Raum bislang wenig systematisch aufbereitet ist, bezieht sich Seipold auf den britischen medienpädagogischen und erziehungswissenschaftliche Diskussionsstand. Sie ist Mitglied der London Mobile Learning Group (LMLG) und diskutiert in inrem Beitrag diverse Erkenntnisse hinsichtlich möglicher Konsequenzen für die Theorieentwicklung, Praxisforschung und die Implementierung von Mobilem Lernen in formalen schulischen Kontexten. Die Autorin bündelt zudem die britische und deutschsprachige medienpädagogische und erziehungswissenschaftliche Mobile-Learning-Diskussion und erstellt eine eigene Systematisierung. Um die strukturellen Aspekte der Mobile-Learning-Diskussion nachvollziehen zu können, verweist sie auf ausgewählte Theorien (z.B. Activity Theorie nach Yrjö Engeström, Conversational Framework nach Diana Laurillard). Die London Mobile Learning Group bezieht sich zunächst auf diese Theorien, entwickelt jedoch ein eigenes Modell der "Sozio-kulturellen 
Ökologie Mobilen Lernens» sowie einen eigenen Analyserahmen. Das Modell sieht ein Zusammenspiel sozio-kultureller und technologischer Strukturen, Handlungskompetenzen des Lernenden und ihrer kulturellen Praktiken vor und fokussiert die Lebenswelt des Lernenden. Seipold betrachtet daraufhin das Thema hinsichtlich einer Implementierung von Mobile Learning in der Schulpraxis und verweist darauf, dass sich trotz aller Systematisierungsversuche weiterhin Unklarheiten und Widersprüche sowohl in der Theorie als auch in der Praxis zeigen. Daher bedarf es vielmehr einer kritisch reflektierten Entscheidung für oder gegen den Einsatz von mobilen Endgeräten zur Unterstützung des jeweiligen Lehr- und Lernszenarios.

Das Autorenteam Specht, Kalz und Börner diskutiert aktuelle Trends und Perspektiven im Bereich der mobilen und ubiquitären Lerntechnologien, welche die klassischen Konzepte von Mobile Learning erweitern. Anhand dieser Trends werden die Konsequenzen für das didaktische Design und Lernkonzepte diskutiert. Sie nehmen dazu auf die Ergebnisse der Studie des Networks of Excellence STELLAR Bezug. 2010 wurden international renommierte Experten zum Thema mobiles und allgegenwärtiges Lernen interviewt. Die Experten wurden zunächst gebeten, Ideen zu möglichen Problemfeldern zu benennen. Die Aussagen wurden daraufhin zu Themenfeldern geclustert (z.B. Lernzugang, Lerneinschränkungen usw.). Die Cluster wurden von den Experten hinsichtlich der Umsetzbarkeit und Dringlichkeit gewichtet und ausgewertet. Eine weitere Aufgabe bestand darin, Technologietrends (Smartphones als persönliche Lernportale, ortsbasierte und kontextsensitive Lerntechnologie, Mobile Augmented Reality usw.) zu identifizieren. Die Autoren gehen in ihrem Beitrag ausführlich auf diese Technologietrends ein, um abschliessend Zukunftsperspektiven mobilen Lernens abzuleiten. Sie fokussieren dabei insbesondere die Perspektive des Lernenden und verweisen darauf, dass eine sinnvolle Erweiterung und Integration von Informationen bei der Gestaltung von Lehr- und Lernarrangements vorzunehmen sei. Darüber hinaus sei die Berücksichtigung der jeweiligen Nutzungssituation sowie eine sinnvolle Abstimmung verschiedener Lernaktivitäten bzw. die Koppelung verschiedener Lernangebote notwendig.

Im letzten Beitrag des einführenden Blocks zum Thema Lernen, Arbeiten und Forschen mit Mobile Learning stellt Matthias Rohs den Aspekt des informellen Lernens im Kontext des Mobile Learning heraus. Er weist darauf hin, dass das informelle Lernen nicht ausschliesslich ausserhalb formal organisierter Strukturen zum Einsatz kommt, sondern auch in formellen Lernszenarien realisierbar ist. Daraufhin wird die Frage aufgeworfen, ob und wie 
eine Förderung informellen Lernens überhaupt möglich ist. Zunächst wird jedoch ausgehend von einer begrifflichen und theoretischen Einordnung das informelle mobile Lernen erläutert, um anschliessend die Potenziale aus lerntheoretischer und didaktischer Perspektive betrachten zu können. Um den praxisnahen Bezug im Kontext von Lehr- und Lernszenarien herzustellen, werden daraufhin praktische Anwendungsformen anhand konkreter Beispiele dargestellt. Der Autor beleuchtet abschliessend kritische Aspekte des informellen mobilen Lernens und verweist auf strukturelle, politische Implikationen. Insbesondere die Entgrenzung zwischen beruflichen und privaten Kontexten sollte nach Meinung des Autors kritisch reflektiert werden. Die Entwicklung einer brauchbaren Didaktik, für den theoretischen und praktischen Einbezug befindet sich laut Rohs derzeit noch im Anfangsstadium.

\section{Teil 2: Mobile Learning an Hochschulen}

Das Autorenteam Wegener, Prinz und Leimeister berichtet im ersten Beitrag des Themenblocks Mobile Learning an Hochschulen über Erfahrungen ihres Pilotprojekts: Mobiles Lernen unter Einbezug von Tablet-PCs in universitären Massenveranstaltungen an der Universität Kassel. Auf Basis des UTAUT-Modells bzw. der Online-Befragung wurden wissenschaftliche Erkenntnisse hinsichtlich der Einflussfaktoren auf die Nutzungsintention und die Lernendenzufriedenheit während der Präsenzveranstaltung als auch ausserhalb dieser erhoben und evaluiert. Da bislang keine vergleichbaren Studien hinsichtlich der Einflussfaktoren auf die Nutzungsintensität des selbst gesteuerten mobilen Lernens (jederzeit und überall, ohne direkte Kontrolle des Lehrenden) im Vergleich zu präsenzgebundenem mobilen Lernprozessen (Bereicherung der Präsenzveranstaltung durch mobile Endgeräte und Drahtlosnetzwerke) vorliegen, verfolgte die onlinebasierte Umfrage das Ziel, die Unterschiede zu analysieren. Das Autorenteam ging zudem der Frage nach, wie der mangelnden Interaktivität im Hörsaal begegnet werden kann und ob die Art des genutzten Endgerätes (z.B. iPad, Laptop, Smartphone) einen Einfluss auf die Nutzung und Zufriedenheit hat. Die Ergebnisse bzw. signifikante Unterschiede lassen sich wie folgt zusammenfassen: Sind es im Hörsaal soziale Faktoren und unterstützende Bedingungen, die beim mobilen Lernen eine Rolle spielen, ist es beim selbst gesteuerten Lernen vielmehr der individuell empfundene Mehrwert. Da die Unterschiede zwischen dem präsenzgebundenen und dem selbst gesteuerten mobilen Lernen sehr gross sind, empfehlen die Autoren hinsichtlich der Auswahl von 
Lehr- und Lernszenarien eine differenzierte Herangehensweise sowie eine gezielte Auswahl mobiler Endgeräte.

Die Autoren Rensing und Tittel beschreiben Potenziale, Herausforderungen, technische Möglichkeiten sowie Beispiele des situierten mobilen Lernens. Das situierte Lernen ermöglicht das Lernen anhand eines konkreten Anwendungsfalls, die Lernsituation sollte im besten Fall so gestaltet sein, dass sich Lern- und Anwendungssituation möglichst ähnlich sind. Um sich der Thematik zu nähern, werden zunächst einige Aspekte und Methoden des situierten Lernens thematisiert, dabei spielen sowohl Unterscheidungsmerkmale als auch Gemeinsamkeiten hinsichtlich des kontextualisierten Lernens eine grosse Rolle. Die Ergebnisse werden von den Autoren in einem Modell zusammengeführt. Abschliessend werden einige Lehr- und Lernszenarien vorgestellt und kritisch diskutiert. Während die folgenden zwei Beispiele dem situierten Konzept des Mobilen Lernens folgen, bei dem ein unmittelbarer Bezug zwischen der physischen Umgebung des Lernenden und den Lerninhalten und dem Lernprozess hergestellt wird (Bsp.: Situiertes, aktivierendes Lernen mittels eines Wikis im Bauingenieurwesen; Situierter Zugriff auf Lernmaterialien in der Landtechnik), folgt das letzte Beispiel dem Konzept des kontextualisierten, nicht situierten Mobilen Lernens, bei dem kein unmittelbarer Bezug zur physischen Umgebung des Lernenden hergestellt wird, sondern zu Textabschnitten innerhalb eines Lehrbuchs (Bsp.: Kontextualisierte Bereitstellung von Lernmedien in Ergänzung zum Lehrbuch).

Insbesondere das Thema Augmented Reality wird sich in den nächsten Jahren im Bereich des mobilen Lernens bzw. der Nutzung mobiler Endgeräte voraussichtlich durchsetzen. Viele Applikationen ermöglichen bereits heute die Erweiterung realer Welten mittels der Anzeige kontextsensitiver Informationen. Peter A. Henning stellt in seinem Beitrag die Frage hinsichtlich einer realen Abbildung der Lebenswirklichkeit von Studierenden. Diese nutzen seiner Meinung nach ganz selbstverständlich das Smartphone, um sich über Sachverhalte zu informieren oder sich räumlich zu orientieren. Seit einigen Jahren werde an Hochschulen die Zufriedenheit von Studierenden in der Studieneingangsphase durch zusätzliche Angebote wie z.B. MentoringProgramme oder Orientierungswochen gefördert, um den Studienerfolg zu optimieren. Henning stellt in seinem Beitrag das Forschungsprojekt STOBAR vor. Studierende werden während der Studieneingangsphase neben traditionellen Orientierungsveranstaltungen zusätzlich durch AugmentedReality-Angebote unterstützt, den Prozess bezeichnet der Autor als «student 
onboarding». Als technisches Hilfsmittel dienen die Smartphones der Studierenden. Eine App vermittelt a) räumliche Informationen mit b) zeitlichen Bezügen sowie c) weiteren Informationen. Der Autor erläutert des Weiteren technische Details und Herausforderungen sowie diverse Potenziale des Projekts und verweist auf perspektivische Überlegungen für den Bereich Hochschule.

In ihrem Beitrag «Urheber- und datenschutzrechtliche Fragen des Mobile Learning» diskutieren die Autoren Forgó und Heinemeyer mögliche rechtliche Fragestellungen und Probleme, die an Hochschulen als Anbieter mobiler Lehr- und Lernangebote herangetragen werden könnten. Neben den rechtlichen Bedingungen werden auch Rechtsfolgen etwaiger Verstösse gegen die einschlägigen Normen erläutert, aus denen sowohl finanzielle als auch organisatorische Folgen resultieren können. Abschliessend sprechen sie die Empfehlung aus, hinsichtlich der Nutzung von Mobile LearningSystemen auch in Bezug auf personenbezogene Daten sensibilisiert vorzugehen. Die Systeme sollten vor Betriebsaufnahme einer rechtlichen Überprüfung unterzogen werden.

\section{Teil 3: Mobile Learning in Unternehmen}

Im dritten Teil des Sammelwerks wird das Thema Mobile Learning aus dem Blickwinkel von Unternehmen bzw. entlang der Nahtstelle Wissenschaft und Unternehmen betrachtet. Die Autorinnen Gloerfeld und Sieber stellen in inrem Beitrag Forschungsergebnisse mobiler Lernszenarien in der beruflichen Bildung vor. Das Projekt wurde vom Bundesministerium für Bildung und Forschung (BMBF) gefördert und von der Forschungsgruppe Mobile Learning an der FernUniversität in Hagen koordiniert und wissenschaftlich begleitet. Untersucht und bewertet wurden neben der Konzeption, der praxisnahen Erprobung und Bewertung zweier zielgruppenspezifischer mobiler Lernanwendungen auch Lernszenarien für verschiedene Lernanforderungen. Die Ergebnisse im vorliegenden Artikel stammen aus zwei von drei Teilprojekten (das dritte Teilprojekt wird in einem separaten Artikel vorgestellt). Als Zielgruppe wurden Berufskraftfahrer (1) und Auszubildende der Elektrotechnik (2) herangezogen. Für die erste Zielgruppe wurden Inhalte zum Arbeits- und Gesundheitsschutz entwickelt, welche auf mobilen Endgeräten bearbeitet werden sollten. Die zweite Zielgruppe wurde hinsichtlich des Einsatzes mobiler Endgeräte im Kontext Berufsschule und Arbeitsplatz evaluiert, im Blickpunkt stand die sinnvolle und effiziente Nutzung der mobilen

Endgeräte, die Aneignung von Inhalten an verschiedenen Lernorten sowie 
die Entwicklung bildungsortübergreifender Lernaktivitäten. Insbesondere der Transfer der verschiedenen Lernformen sowie die Unterstützung einer kontinuierlichen Kommunikationskultur während der Ausbildung wurde evaluiert und ist zugleich bereits Teil einer abschliessenden Empfehlung des Autorenteams. Die Einführung neuer mobiler Lernszenarien ist bei den beiden Zielgruppen auf positive Resonanz gestossen, im Detail müssen aber für einen dauerhaften Einsatz einige Aspekte wie z.B. die Berücksichtigung des Mehraufwands und die dauerhafte Pflege von Inhalten und Systemen bedacht werden. Insbesondere der Einbezug aller beteiligten Personen ist nach Einschätzung des Autorenteams von grosser Bedeutung.

Im nächsten Beitrag wird das dritte Teilprojekt aus der zuvor vorgestellten Studie beschrieben. Es handelt sich dabei um die Realisierung mobiler Lernmöglichkeiten in der Automobilindustrie bei der Daimler AG. Der Autor Volker Engert ist ebenfalls in der Forschungsgruppe Mobile Learning tätig, die Herangehensweise ist daher identisch mit der bereits im Beitrag der Autoren Gloerfeld und Sieber erläuterten wissenschaftlichen Studie. Im vorliegenden Fall wurde ein Produktionslernsystem entwickelt, um die Dokumentation von Arbeitsprozessen sowie die Sicherung von Erfahrungswissen zu ermöglichen. Der Autor erläutert im vorliegenden Beitrag Einsatzmöglichkeiten mobiler Endgeräte für die Mitarbeiterqualifizierung unter Einbezug des Produktionslernsystems. Dieses dient dazu, Arbeitsprozesse zu dokumentieren und anderen zur Verfügung zu stellen, zugleich unterstützt der Einsatz mobiler Endgeräte den Prozess der arbeitsplatznahen Qualifizierung. Der Autor erwartet, dass sich im Bereich der Aus- und Weiterbildung die handlichen Tablet-PCs durchsetzen werden.

Im Beitrag von Keist und Gissler wird ein weiteres Beispiel aus der Unternehmenspraxis präsentiert. Die Autoren zeigen, wie Blended Learning und mobiles Lernen bei der Schweizerischen Post an Bedeutung zunimmt. Auch werden Spannungsfelder in Bezug auf Lern- und Arbeitsprozesse und deren Herausforderungen benannt. Um Lösungswege aufzuzeigen, wurde zunächst eine Evaluation eines berufsbegleitenden Blended-LearningLehrgangs vorgenommen. Die Autoren setzen sich kritisch mit den bisherigen Angeboten der Schweizerischen Post im E-Learning Bereich auseinander und reflektieren die E-Learning-Angebote hinsichtlich einer Übertragbarkeit auf mobile Anwendungen. Da dies nicht 1:1 realisierbar ist, soll bei der Schweizerischen Post langfristig ein entsprechendes Konzept erarbeitet werden, wobei erste entsprechende Konzeptideen bereits vorliegen. 
Im nächsten Beitrag wird ebenfalls ein Beispiel zum mobilen Lernen in der Aus- und Weiterbildung eines Unternehmens - der Lufthansa AG - präsentiert. Das Autorenteam Harriehausen-Mühlbauer und Sonne stellt ihr Lufthansa Privacy-Quiz vor, welches Mobile Gaming auf Smartphones ermöglicht. Zusammen mit der TU Dortmund wurde eine Lern-App für das Lufthansa-Management zum Thema Data Privacy entwickelt. Das Spielprinzip basiert auf dem «Wer wird Millionär?»-Quiz und dient dem Wissensaufbau zum Thema Datenschutz und Datensicherheit. Die Autoren betonen, dass seitens des Unternehmens eine entsprechende Lernkultur geschaffen werden muss, um das Lernen mit mobilen Endgeräten zu unterstützen. Da die technologische Unterstützung von Lernprozessen von Mitarbeitern der Lufthansa AG bereits seit Jahrzehnten im Unternehmen verankert ist, sind die Mitarbeiter/innen neuen Entwicklungen gegenüber positiv aufgeschlossen. Im Verlauf des Beitrags werden technische Aspekte diskutiert, die Einbindung eines Autorentools als auch diverse Aspekte hinsichtlich der App-Programmierung. Die Autoren planen die Entwicklung weiterer Mini Games und sehen im Bereich des Game-Based-Learning viel Potenzial.

Im letzten Beitrag von Onno Reiners wird aufgezeigt, wie die technologische Entwicklung und Verbreitung von mobilen Endgeräten der betrieblichen Weiterbildung neue Anwendungsbereiche eröffnet. Der Autor stellt Lernlösungen für iPhone \& Co vor, welche weniger das Lernen und Qualifizieren fokussieren, sondern vielmehr die Aspekte Informieren, Ad-hoc-Lernen und kontextbezogenes Lernen. Eingangs geht der Autor auf diverse Veröffentlichungen zum Lernen mit digitalen Medien ein, diskutiert anschliessend die Themen Entgrenzung, Kontextualisierung und Beziehungsstiftung und benennt Besonderheiten des mobilen Lernens. Abschliessend präsentiert und diskutiert er diverse Lernlösungen für mobiles Lernen in der betrieblichen Weiterbildung.

\section{Gesamtbetrachtung}

Obwohl das Thema Mobile Learning in der Bildungslandschaft seit einigen Jahren diskutiert und erprobt wird, zeigt sich bei der Recherche nach entsprechenden Publikationen, dass es nur vereinzelte Artikel und Essays gibt. Eine zusammenfassende Darstellung des Themas Lernen mit mobilen Endgeräten über die Bildungssektoren hinweg fehlt.

Der vorliegende Sammelband der Herausgeberinnen de Witt und Sieber füllt diese Lücke, indem er einen umfangreichen und aktuellen Überblick zum Thema Lernen mit mobilen Endgeräten bietet. Sowohl die theoretische An- 
näherung an das Thema als auch konkrete Best-Practice-Beispiele aus Hochschulen und Unternehmen werden berücksichtigt. Es wird allerdings deutlich, dass das Thema Mobile Learning bislang wesentlich häufiger im Hochschulkontext diskutiert (und realisiert) wird. Mobile Learning in Unternehmen ist noch eher unbekannt bzw. womöglich nicht umfassend dokumentiert.

Die Beiträge regen dazu an, eigene Konzepte und Einsatzszenarien zu reflektieren und ggf. um Szenarien für mobile Endgeräte zu erweitern. Die Herausforderung für E-Akteure besteht darin, das Thema Mobiles Lernen von den bereits etablierten E-Learning Konzepten zu unterscheiden und gleichzeitig Erfahrungswerte zunutze zu machen. So spielen bei der Konzeption von mobilen Lehr- und Lernszenarien ebenfalls Aspekte wie z.B. Zielgruppenanalyse, Transfer von der Theorie in die Praxis usw. eine zentrale Rolle. Auch die Kommunikationskultur und Lernbegleitung der jeweiligen Institution bzw. des Arbeitgebers sowie die Anpassung der Lernangebote und Lernaufgaben an situative und kontextbezogene Anwendungsfelder sind bei der Realisierung zu berücksichtigen.

Anke Petschenka 